\title{
Urine cell-based DNA methylation classifier for monitoring bladder cancer
}

\author{
Antoine G. van der Heijden ${ }^{1 \dagger}$, Lourdes Mengual ${ }^{2,6^{*}+} \mathbb{D}$, Mercedes Ingelmo-Torres ${ }^{2}$, Juan J. Lozano ${ }^{3}$, \\ Cindy C. M. van Rijt-van de Westerlo' ${ }^{1}$, Montserrat Baixauli ${ }^{2}$, Bogdan Geavlete ${ }^{4}$, Cristian Moldoveanud4, \\ Cosmin Ene ${ }^{4}$, Colin P. Dinney ${ }^{5}$, Bogdan Czerniak ${ }^{5}$, Jack A. Schalken', Lambertus A. L. M. Kiemeney ${ }^{1}$, Maria J. Ribal², \\ J. Alfred Witjes ${ }^{1}$ and Antonio Alcaraz ${ }^{2}$
}

\begin{abstract}
Background: Current standard methods used to detect and monitor bladder cancer (BC) are invasive or have low sensitivity. This study aimed to develop a urine methylation biomarker classifier for BC monitoring and validate this classifier in patients in follow-up for bladder cancer (PFBC).

Methods: Voided urine samples $(N=725)$ from BC patients, controls, and PFBC were prospectively collected in four centers. Finally, 626 urine samples were available for analysis. DNA was extracted from the urinary cells and bisulfite modificated, and methylation status was analyzed using pyrosequencing. Cytology was available from a subset of patients $(N=399)$. In the discovery phase, seven selected genes from the literature (CDH13, CFTR, NID2, SALL3, TMEFF2, TWIST1, and VIM2) were studied in 111 BC and 57 control samples. This training set was used to develop a gene classifier by logistic regression and was validated in 458 PFBC samples (173 with recurrence).

Results: A three-gene methylation classifier containing CFTR, SALL3, and TWIST1 was developed in the training set (AUC 0.874). The classifier achieved an AUC of 0.741 in the validation series. Cytology results were available for 308 samples from the validation set. Cytology achieved AUC 0.696 whereas the classifier in this subset of patients reached an AUC 0.768. Combining the methylation classifier with cytology results achieved an AUC 0.86 in the validation set, with a sensitivity of $96 \%$, a specificity of $40 \%$, and a positive and negative predictive value of 56 and $92 \%$, respectively.

Conclusions: The combination of the three-gene methylation classifier and cytology results has high sensitivity and high negative predictive value in a real clinical scenario (PFBC). The proposed classifier is a useful test for predicting $\mathrm{BC}$ recurrence and decrease the number of cystoscopies in the follow-up of $\mathrm{BC}$ patients. If only patients with a positive combined classifier result would be cystoscopied, 36\% of all cystoscopies can be prevented.
\end{abstract}

Keywords: Cytology, Biomarkers, Bladder cancer, DNA methylation, Non-invasive diagnosis, Urine

\section{Background}

Seventy to $80 \%$ of patients with bladder cancer (BC) present with non-muscle-invasive tumors, either confined to the mucosa [stage Ta and carcinoma in situ (CIS)] or submucosa (stage T1). Based on clinical and

\footnotetext{
* Correspondence: LMENGUAL@clinic.cat

${ }^{\dagger}$ Antoine G. van der Heijden and Lourdes Mengual contributed equally to this work.

${ }^{2}$ Laboratory and Department of Urology, Hospital Clinic of Barcelona,

IDIBAPS, University of Barcelona, Barcelona, Spain

${ }^{6}$ Hospital Clínic de Barcelona, Centre de Recerca Biomèdica CELLEX, office

B22, C/Casanova, 143, 08036 Barcelona, Spain

Full list of author information is available at the end of the article
}

pathological characteristics, non-muscle-invasive bladder cancer (NMIBC) patients can be classified into three different prognostic groups [1]. A minority of patients (20-30\%) have low-risk tumors with a recurrence rate of $20-30 \%$, without progression. The second and also the largest group, the intermediate-risk group, consists of patients who frequently develop a non-muscle-invasive recurrence $(40-60 \%)$ but seldom progress to muscle-invasive disease. Finally, a small group of patients has a relatively aggressive NMIBC at presentation. The 5-year recurrence rate in this group is as high as $68 \%$ despite maximum intravesical treatment.

(c) The Author(s). 2018 Open Access This article is distributed under the terms of the Creative Commons Attribution 4.0 International License (http://creativecommons.org/licenses/by/4.0/), which permits unrestricted use, distribution, and 
Furthermore, up to $34 \%$ of these high-risk patients will develop muscle-invasive bladder cancer (MIBC) [2]. For this reason, an intensive follow-up schedule is mandatory in patients with intermediate- or high-risk NMIBC.

The follow-up schedule consists of urethrocystoscopy and urine cytology. Depending on the patient's risk profile, the European Association of Urology guidelines recommend up to 15 urethrocystoscopies during the first 5 years of follow-up [3].

Urethrocystoscopy is considered the gold standard, but is invasive, expensive, and moreover misses up to $15 \%$ of the papillary and up to $30 \%$ of the flat recurrences $[4,5]$. Urine cytology, on the other hand, has a high specificity (SP) but lacks sensitivity (SN) especially in low-risk tumors [6]. Additionally, the interobserver and intraobserver reproducibility of cytology is poor [7]. Recently, several non-invasive methods, NMP-22, bladder tumor antigen, and UroVysion FISH, have shown to help increase the sensitivity of urine cytology. However, due to limited specificity or sensitivity, the markers proposed to date have not been widely adopted in daily clinical practice. Therefore, there is a clear clinical need to find reliable markers to monitor the recurrence in NMIBC [8].

DNA methylation has been recognized to be important in developmental biology and cancer etiology in general [9]. DNA methylation occurs principally at CpG dinucleotides. These $\mathrm{CpG}$ dinucleotides are distributed throughout the genome, and the majority is normally methylated. Some regions in the genome have a high CpG density and are called CpG islands. Hypermethylation of normally unmethylated $\mathrm{CpG}$ islands in the promoter regions of tumor suppressor genes represses its transcription in human tumors $[9,10]$. Therefore, aberrant DNA methylation is a potential biomarker for diagnosis, prognosis, and monitoring of disease after therapy [11]. Recently, it was shown that the combination of SOX1, IRAK3, and L1-MET provides better resolution than cytology and cystoscopy in the detection of early recurrence [12]. The objective of the present study is to investigate whether a set of methylation markers can lead to the development of a voided urine test that predicts tumor presence and may be used to stratify BC patients according to their risk of recurrence, thus allowing the reduction of the number of cystoscopies in the follow-up of BC.

\section{Methods}

\section{Patients and clinical samples}

After Institutional Review Board approval and obtaining patients' informed consents, we prospectively collected freshly voided urine samples from $\mathrm{BC}$ patients, controls, and patients in follow-up for bladder cancer (PFBC) at four different centers [Hospital Clínic of Barcelona (Spain); Radboud University Medical Center in Nijmegen (The Netherlands); St. John Emergency Hospital, Bucharest (Romania); MD Anderson Cancer Center, Houston, Texas, (USA)], from October 2010 to February 2012. Participating centers were asked to collect and prepare the cell pellet by urine centrifugation and freeze them for a final processing at the Hospital Clinic of Barcelona or Radboud University Medical Center, Nijmegen. We took a two-stage approach with a discovery phase (or training set) and a validation phase (or testing set) (Additional file 1: Figure S1). In the discovery phase, the inclusion criteria for the cases were patients of both sexes, 18 years of age or older, and patients with histopathological confirmation of $\mathrm{BC}$ at any grade or stage. Without being mandatory, we recommended patients to have cytology at cystoscopy or during the period between cystoscopy and surgery. Patients with a prior endovesical chemotherapeutic or immunotherapeutic treatment could be included. The exclusion criteria were the absence of histological confirmation of $\mathrm{BC}$ and patients with other urological malignancies (prostate, kidney, urinary tract tumors). The inclusion criteria for the controls were patients of both sexes, 18 years of age or older, and with non-malignant urologic pathology (infection, lithiasis, urinary incontinence, BPH) or non-urologic pathology. The exclusion criterion for controls was a histological confirmation of any urological malignancy.

The validation phase was designed as a cross-sectional study including $\mathrm{PFBC}$, i.e., the indicated population for the test in daily clinical practice. For efficiency reasons, we oversampled patients with a recurrence because we focused on sensitivity instead of specificity (see also the results in the "Reducing the number of follow-up cystoscopies by using the three-gene methylation/cytology combined classifier" section). PFBC with a prior endovesical chemotherapeutic or immunotherapeutic treatment could be included. Without being mandatory, we recommended PFBC to have cytology at cystoscopy. The exclusion criterion was a histological confirmation of any other urological malignancy.

A total number of 725 voided urine samples were prospectively collected by the four participating institutions. From the total number of urines collected, 99 (13\%) were excluded from the study because of technical problems during the sample collection, storage, or analysis. Finally, 626 urines were used: 111 from BC patients and 57 from controls for the discovery phase and 458 from PFBC (of whom 173 had a recurrence) for the validation phase (Tables 1 and 2). The grade and stage of the tumors were determined according to WHO 2004 criteria and TNM 2002 classification, respectively [13, 14].

Tumors were classified according to their risk of recurrence and progression into three categories: high-risk 
Table 1 Clinicopathological and demographic characteristics of the study population classified by the study phase

\begin{tabular}{|c|c|c|}
\hline & Discovery phase & Validation phase \\
\hline & Training set & Testing set \\
\hline & $N$ bladder cancer (\%) & N R-PFBC (\%) \\
\hline \multicolumn{3}{|l|}{ Gender } \\
\hline Male & $86(77)$ & $135(78)$ \\
\hline Female & $25(23)$ & $38(22)$ \\
\hline \multicolumn{3}{|l|}{ Age } \\
\hline Mean & 72 & 68 \\
\hline Range & $39-98$ & $26-99$ \\
\hline \multicolumn{3}{|l|}{ Stage and grade } \\
\hline Tis & $7(6)$ & $13(8)$ \\
\hline Ta LG & $26(23)$ & $61(35)$ \\
\hline $\mathrm{Ta} H \mathrm{HG}$ & $11(10)$ & $2012)$ \\
\hline $\mathrm{T} 1 \mathrm{LG}$ & $20(18)$ & $35(20)$ \\
\hline $\mathrm{T} 1 \mathrm{HG}$ & $22(20)$ & $44(25)$ \\
\hline$>\mathrm{T} 2 \mathrm{LG}$ & $1(1)$ & - \\
\hline$>\mathrm{T} 2 \mathrm{HG}$ & $24(22)$ & - \\
\hline \multirow[t]{2}{*}{ Subtotals } & 111 & 173 \\
\hline & N control (\%) & \\
\hline \multicolumn{3}{|l|}{ Gender } \\
\hline Male & $29(51)$ & - \\
\hline Female & $28(49)$ & - \\
\hline \multicolumn{3}{|l|}{ Age } \\
\hline Mean & 60 & - \\
\hline Range & $22-82$ & - \\
\hline \multicolumn{3}{|l|}{ Urinary condition } \\
\hline $\mathrm{BPH}$ & $11(19)$ & - \\
\hline Urolithiasis & $13(23)$ & - \\
\hline Incontinence & $2(4)$ & - \\
\hline Benign bladder disease & $1(2)$ & - \\
\hline Urinary tract infections & $12(21)$ & - \\
\hline Non-urological diseases & $18(32)$ & - \\
\hline \multirow[t]{2}{*}{ Subtotals } & 57 & - \\
\hline & & N NR-PFBC (\%) \\
\hline \multicolumn{3}{|l|}{ Gender } \\
\hline Male & - & $217(76)$ \\
\hline Female & - & $68(24)$ \\
\hline \multicolumn{3}{|l|}{ Age } \\
\hline Mean & - & 69 \\
\hline Range & - & $26-92$ \\
\hline \multicolumn{3}{|c|}{ Stage and grade previous TURBT } \\
\hline Tis & - & $21(7)$ \\
\hline Ta LG & - & $100(35)$ \\
\hline Ta HG & - & $53(19)$ \\
\hline
\end{tabular}

Table 1 Clinicopathological and demographic characteristics of the study population classified by the study phase (Continued)

\begin{tabular}{cll}
\hline & $\begin{array}{l}\text { Discovery phase } \\
\text { Training set }\end{array}$ & $\begin{array}{l}\text { Validation phase } \\
\text { Testing set }\end{array}$ \\
\hline T1 LG & - & $22(8)$ \\
T1 HG & - & $82(29)$ \\
T2 HG & - & $3(1)$ \\
Tx LG & - & $2(1)$ \\
Tx HG & - & $2(1)$ \\
Subtotals & - & 285 \\
Total & 168 & 458
\end{tabular}

LG low-grade, HG high-grade, TURBT transurethral resection bladder tumor, $B P H$ benign prostate hyperplasia, CIS/Tis carcinoma in situ, $B C$ bladder cancer, $R$-PFBC recurrent patients in follow-up for bladder cancer, NR-PFBC nonrecurrent patients in follow-up for bladder cancer

(HR) NMIBC (any of the following: T1, HG/G3 tumors, or CIS), non-high-risk (nHR) NMIBC (all the other cases of NMIBC), and muscle-invasive bladder cancer (MIBC) (T2-4). None of the included patients had an upper urinary tract tumor.

\section{Urine sample processing}

Urine samples were collected before cystoscopy, the day before the transurethral resection of the bladder tumor (TURBT), or the day before cystectomy. From all patients and controls, only one single sample was included.

\section{For urine cytology}

Urine cytology was performed according to Papanicolaou staining and evaluated by expert pathologists in each center blinded to the patient's clinical history. The results were considered as positive or negative. No central cytology review was performed.

\section{For methylation studies}

Voided urine samples (50 to $100 \mathrm{ml}$ ) were collected in sterile containers containing $4 \mathrm{ml}$ of $0.5 \mathrm{M}$ EDTA, $\mathrm{pH}$ 8.0. Urines were immediately stored at $4{ }^{\circ} \mathrm{C}$ and processed within the next $24 \mathrm{~h}$. The samples were centrifuged at $1000 \times g$ for $10 \mathrm{~min}$, at $4{ }^{\circ} \mathrm{C}$. The cell pellets were frozen at $-80{ }^{\circ} \mathrm{C}$.

\section{DNA isolation, bisulfite treatment, and PCR}

DNAs from the urinary cell pellets were extracted using QIAamp DNA Mini Kit (Qiagen) according to the manufacturer's instructions and quantified with a NanoDrop1000 (NanoDrop Technologies, Wilmington, DE, USA). DNA extraction was performed in each center except for Bucharest, whose cell pellets were sent in dry ice to the Radboud University Medical Center, Nijmegen (The Netherlands) for DNA extraction.

One microgram of genomic DNA was used for the bisulfite modification using EpiTect Bisulfite kit (Qiagen, 
Table 2 Clinicopathological and demographic characteristics of the study population classified by the participating center

$$
\text { Hospital Clinic Barcelona Radboud University Saint John Emergency MD Anderson Cancer Total }
$$
Medical Center, Nijmegen Clinical Hospital Bucharest Center Houston

Discovery phase Bladder cancer urine samples

Stage

Tis 5

Ta 14

19

$\mathrm{T1}$

\section{9}

$>\mathrm{T} 2$

9

Grade

$$
\text { HG }
$$

20

27

Subtotal

$$
47
$$

Control urine samples

$\begin{array}{ll}\text { BPH } & 8 \\ \text { Urolithiasis } & 13 \\ \text { Incontinence } & 1 \\ \begin{array}{l}\text { Benign bladder } \\ \text { disease }\end{array} & 4 \\ \begin{array}{l}\text { Urinary tract } \\ \text { infection }\end{array} & 1 \\ \begin{array}{l}\text { Non-urological } \\ \text { diseases }\end{array} & 6 \\ \text { Subtotal } & 33\end{array}$

Subtotal

Validation phase R-PFBC URINE SAMPLES

Stage
Tis
Ta
$\mathrm{T} 1$

Grade

$$
\text { LG }
$$

$$
\text { HG }
$$

Subtotal

2
13
4

$2-1$

15

2

3

$-$

NR-PFBC urine samples

Stage previous TURBT

Tis
$\mathrm{Ta}$
$\mathrm{Ta}+\mathrm{CIS}$
$\mathrm{T} 1$
$\mathrm{~T} 1+\mathrm{CIS}$
$\mathrm{T} 2$
$\mathrm{Tx}$

14
5
19

8

\section{4}

Grade previous TURBT

\begin{tabular}{clllll} 
LG & 34 & 35 & 38 & 19 & 126 \\
HG & 52 & 43 & 29 & 35 & 159 \\
Subtotal & 86 & 78 & 67 & 54 & 285 \\
TOTAL & 185 & 120 & 225 & 96 & 626 \\
\hline
\end{tabular}


Inc.) following the manufacturer's instructions. The modified DNA was eluted with $20 \mu \mathrm{l}$ Tris-HCL $(1 \mathrm{mM}$, $\mathrm{pH}$ 8.0) and stored at $-80{ }^{\circ} \mathrm{C}$ before further processing. Bisulfite modifications were performed in Hospital Clinic of Barcelona, Spain (training set), and in Radboud University Medical Center, Nijmegen, The Netherlands (testing set).

A total of seven DNA methylation markers, i.e., CDH13, CFTR, NID2, SALL3, TMEFF2, TWIST1, and VIM2, were selected from four recently published BC studies [15-18]. The sequences of the primers used to amplify the regions of interest of these genes and the PCR conditions are shown in Additional file 2: Table S1. PCR primers were designed using the PyroMark Assay Design software v2.0 (Qiagen). PCR was performed in a volume of $25 \mu \mathrm{l}$ with $2 \mu \mathrm{l}$ of converted genomic DNA, 0.6 U Ampli Taq Gold 360 DNA polymerase (Thermofisher), $0.8 \mu \mathrm{l}$ of a mix of Primer-F and biotinylated Primer-R at $10 \mu \mathrm{M}, 2 \mu \mathrm{lgCl}_{2} 25 \mathrm{mM}$, and $0.5 \mu \mathrm{l}$ dNTPs $10 \mathrm{mM}$. Amplification was performed according to the following thermocycling conditions: denaturation at $95{ }^{\circ} \mathrm{C}$ for $10 \mathrm{~min}$, followed by 45 cycles of $95{ }^{\circ} \mathrm{C}$ for $30 \mathrm{~s}$, the optimal $\mathrm{Tm}$ for $30 \mathrm{~s}$, and $72{ }^{\circ} \mathrm{C}$ for $1 \mathrm{~min}$; and a final extension at $72{ }^{\circ} \mathrm{C}$ for $7 \mathrm{~min}$. The formation of PCR products with accurate size was confirmed by resolving PCR samples $(1 \mu \mathrm{l})$ by $2 \%$ agarose gel electrophoresis, with visualization by ethidium bromide staining.

\section{Pyrosequencing for quantitative methylation}

Biotin-labeled single-stranded amplicons were isolated from $20 \mu \mathrm{l}$ of the PCR product according to the protocol using the Pyromark Q96 Work Station and pyrosequenced with $0.3 \mu \mathrm{M}$ sequence primer using PSQ96MD System (Biotage AB). Additional file 2: Table S1 shows the sequences of the primer sets used for bisulfite sequencing. The percent methylation for each of the CpGs was calculated using PyroQ CpG Software (Qiagen). The differences in the percentage of methylation were calculated between $\mathrm{BC}$ vs. control and recurrent vs. non-recurrent PFBC (R-PFBC and NR-PFBC, respectively) samples. A high correlation in the methylation percentages of the CPG dinucleotides in the same island was observed (Additional files 3 and 4: Figures S2 and S3). For this reason, hypermethylation was analyzed in all genes at the first CpG dinucleotide present (Additional file 5: Table S2).

\section{Data analysis}

Univariable and multivariable logistic regression analyses were used to examine the associations between $\mathrm{BC}$ and DNA methylation status of urinary sediments. A forward stepwise logistic regression was performed to determine the best classifier between $\mathrm{BC}$ and control samples. The inclusion criterion was $p$ value of $<0.1$. Risk probability of presenting $\mathrm{BC}$ was calculated in the training set. We established cutoff point value $(\geq 0.464)$ allowing $15 \%$ false negatives in the tumor group $(\mathrm{SN}=85 \%)$. In the subset of samples in which cytology results were available, the cutoff point value that yielded $85 \% \mathrm{SN}$ in the training set was 0.688, and in the combined model (methylation test + cytology results), it was 0.617 . If the predicted probability value derived from each classifier in each of the samples was higher than the cutoff point value, the samples were classified for each of the classifiers as tumor sample. The performance of the models was evaluated in a testing set by means of AUROC using pROC R-package [19]. Student's $t$ test was used to evaluate statistical differences in DNA methylation. Statistical significance was established at $p$ value of 0.05 . R-software and SPSS v23.0 were used for calculations.

\section{Results}

Training set

DNA methylation of all seven selected genes was significantly increased in urine sediments from BC patients compared to controls (Fig. 1). To determine the combination of markers capable of detecting $\mathrm{BC}$ in urine sediments with the highest accuracy, we built a model of multiple markers by logistic regression. The best possible biomarker combination based on AUC was provided by the combination of CFTR, SALL3, and TWIST1. This three-gene methylation classifier achieved an $\mathrm{AUC}=0.874$ (Fig. 2a); at a fixed overall $\mathrm{SN}$ of $85 \%$, the classifier provides a SP of $68 \%$. Moreover, the SN of the three-gene methylation classifier increases through the $\mathrm{BC}$ risk groups and grading (Table 3 ).

\section{Testing set}

To examine whether the three-gene methylation $\mathrm{BC}$ diagnostic classifier was able to identify recurrences in a clinical setting, the classifier was validated in an independent multicenter international series of 458 urine sediments from patients in follow-up for bladder cancer (PFBC), of whom 173 had a recurrence. Recurrent PFBC (R-PFBC; $N=173$ ) displays higher percentages of DNA methylation compared with non-recurrent PFBC (NR-PFBC; $N=285$ ) (Fig. 3). $\mathrm{SN}$ of the three-gene methylation $\mathrm{BC}$ classifier increased in the validation series $(\mathrm{SN}=90 \%)$, while SP drops $(\mathrm{SP}=31 \%)$, as evidenced by the ROC curves and AUC value (AUC = 0.741) (Table 3 and Fig. 2a). Figure 4a depicts the risk probabilities derived from the three-gene methylation classifier in R-PFBC and NR-PFBC.

\section{Comparison of test performance with urine cytology}

A total of 399 urine cytologies (91 from the training and 308 from the testing set) were performed. In both 


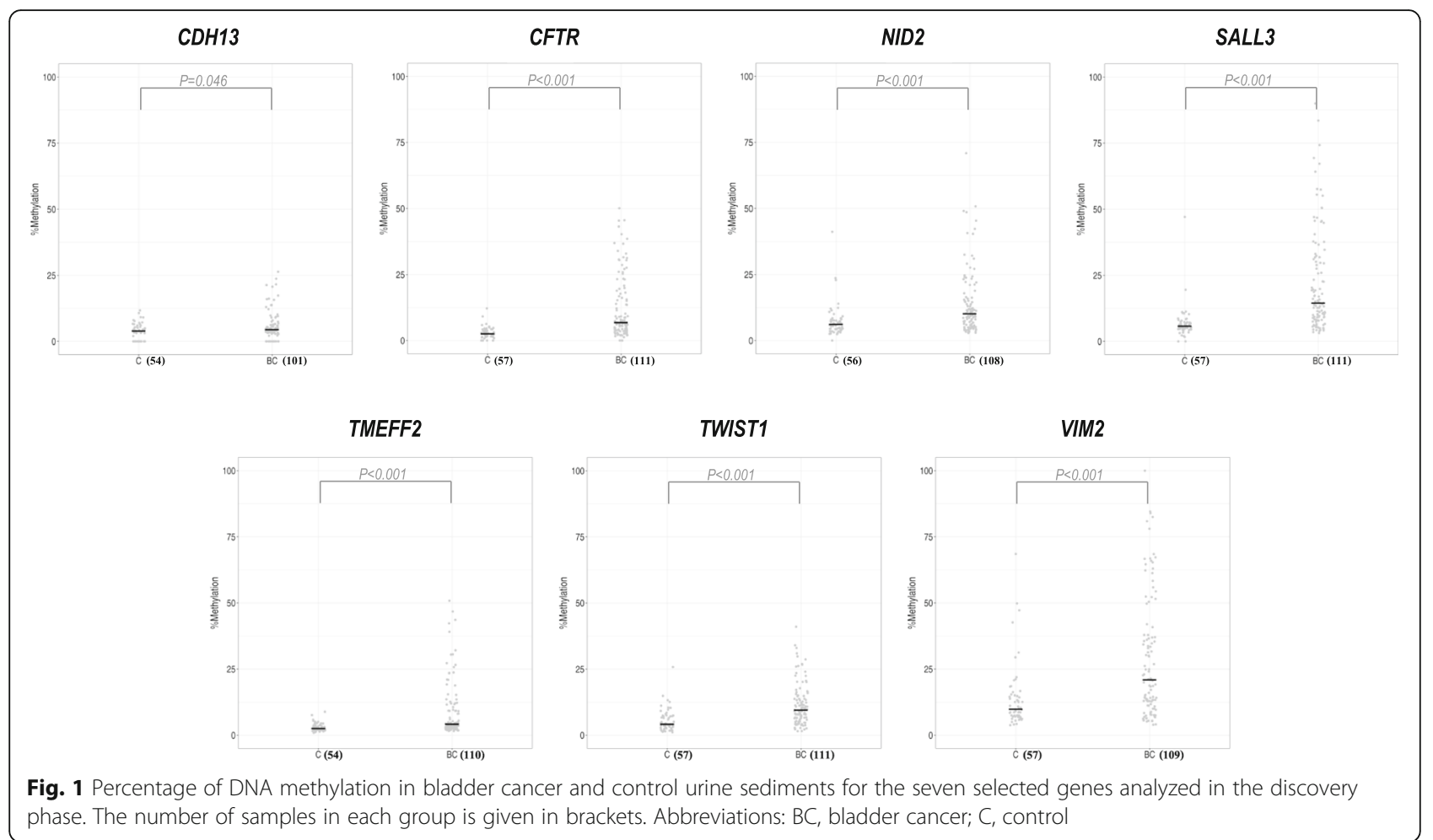

training and testing set, SN of the three-gene methylation classifier (86 and 93\%, respectively) was higher than that of the urine cytology (54 and 46\%, respectively) in this subset of samples (Additional files 6 and 7: Table S3 and Figure S4). In the testing set, this means that $55 \%$ of the recurrences (68 out of 124) were detected by the three-gene classifier but were missed by urine cytology. On the other hand, 12 recurrences were missed by the three-gene classifier of which half was detected by cytology (Additional file 8: Figure S5). Negative predictive value (NPV) is also higher for the three-gene methylation classifier than that of the urine cytology in training (40 and 29\%, respectively) and testing set ( 82 and $69 \%$, respectively). Contrary, positive predictive value (PPV) is higher for the urine cytology than for the methylation classifier in training (98 and 89\%, respectively) and testing set (85 and $50 \%$, respectively). Cytology had a SP of 93 and $94 \%$ while the three-gene methylation classifier achieved a SP, in this subset of samples, of 47 and 27\%, in the training and testing set, respectively. In the testing set, 120 NR-PFBC samples were positive by the three-gene
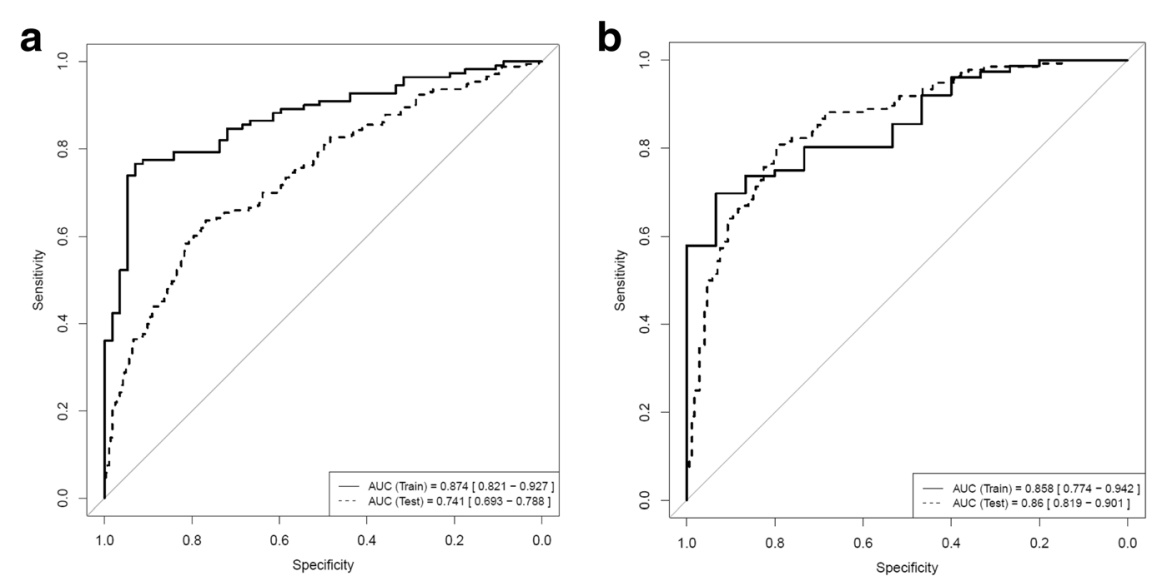

Fig. 2 ROC curves in the training and testing series for (a) the three-gene BC methylation classifier and (b) the combined three-gene methylation/cytology classifier 
Table 3 Diagnostic performance of the three-gene methylation classifier in the training and testing set of samples (at fixed sensitivity of $85 \%$ in the training set)

\begin{tabular}{lll}
\hline & Training set & Testing set \\
\hline Overall & & \\
N samples & $111 \mathrm{BC} / 57 \mathrm{C}$ & $173 \mathrm{R}-\mathrm{PFBC} / 285$ NR-PFBC \\
AUC & 0.874 & 0.741 \\
SN (\%) & 84.68 & 89.6 \\
SP (\%) & 68.42 & 30.53 \\
PPV (\%) & 83.93 & 43.91 \\
NPV (\%) & 69.64 & 82.86 \\
Non-high-risk NMIBC & & \\
N samples & $26 \mathrm{BC} / 57$ C & $61 \mathrm{R}-\mathrm{PFBC} / 285$ NR-PFBC \\
SN (\%) & 73.08 & 88.52 \\
SP (\%) & 68.42 & 30.53 \\
PPV (\%) & 51.35 & 21.43 \\
NPV (\%) & 84.78 & 92.55
\end{tabular}

High-risk NMIBC

$\begin{array}{lll}\text { N samples } & 60 \mathrm{BC} / 57 \mathrm{C} & 112 \mathrm{R}-\mathrm{PFBC} / 285 \text { NR-PFBC } \\ \text { SN (\%) } & 86.67 & 90.18 \\ \text { SP (\%) } & 68.42 & 30.53 \\ \text { PPV (\%) } & 74.29 & 33.78 \\ \text { NPV (\%) } & 82.98 & 88.78\end{array}$

MIBC

$\begin{array}{lll}\text { N samples } & 25 \mathrm{BC} / 57 \mathrm{C} & - \\ \text { SN (\%) } & 92 & - \\ \text { SP (\%) } & 68.42 & - \\ \text { PPV (\%) } & 56.1 & - \\ \text { NPV (\%) } & 95.12 & \\ \text { Low-grade } & & 96 \text { R-PFBC/285 NR-PFBC } \\ \text { N samples } & 47 \mathrm{BC} / 57 \mathrm{C} & 90.62 \\ \text { SN (\%) } & 76.6 & 30.53 \\ \text { SP (\%) } & 68.42 & 30.53 \\ \text { PPV (\%) } & 66.67 & 90.62 \\ \text { NPV (\%) } & 78 & \\ \text { High grade } & & 77 \text { R-PFBC/285 NR-PFBC } \\ \text { N samples } & 64 \text { BC/57 C } & 88.31 \\ \text { SN (\%) } & 90.62 & 30.53 \\ \text { SP (\%) } & 68.42 & 25.56 \\ \text { PPV (\%) } & 76.32 & 90.62 \\ \text { NPV (\%) } & 86.67 & \end{array}$

$L G$ low-grade, $H G$ high-grade, $A U C$ area under the curve, $M I B C$ muscle-invasive bladder cancer, NMIBC non-muscle invasive bladder cancer, NPV negative predictive value, $P P V$ positive predictive value, $S N$ sensitivity, $S P$ specificity, $B C$ bladder cancer, $C$ control, $R$-PFBC recurrent patients in follow-up for bladder cancer, NR-PFBC non-recurrent patients in follow-up for bladder cancer. methylation classifier. Nine of them also had positive urine cytology (Additional file 8: Figure S5). After 1 year, two NR-PFBC with a positive test (who had a negative cytology) had a tumor recurrence.

\section{Combination of the three-gene methylation classifier with urine cytology}

Combining the three-gene methylation classifier and urine cytology results showed an improved diagnostic performance in the training (AUC 0.858) and as well as in the testing set (AUC 0.86) (Fig. 2b). A SN of $96 \%$ and a NPV of $92 \%$ are achieved in the testing set. Of note, in HG tumors, a $100 \%$ SN and NPV are achieved (Additional file 6: Table S3). The risk probabilities derived from the combined classifier in R-PFBC and NR-PFBC are shown in Fig. $4 \mathrm{~b}$.

Reducing the number of follow-up cystoscopies by using the three-gene methylation/cytology combined classifier In our study, we oversampled patients with BC. We therefore cannot directly calculate predictive values from the test results. In the hospitals participating in the study, recurrence is detected in approximately $10 \%$ of all follow-up cystoscopies performed $(90 \%$ of patients previously diagnosed with NMIBC are without recurrence at the time of follow-up cystoscopy). In order to calculate the predictive values that reflect values in real clinical practice, we assumed the distribution of recurrent vs. non-recurrent to be 10 vs. $90 \%$. For this, we multiplied the NR-PFBC samples by 7 . Using the SN and SP that we found in the study, the PPV and NPV in the validation phase become 15 and $99 \%$, respectively. If patients with a negative classifier will not undergo a cystoscopy, this means that more than a third $(\sim 36 \%)$ of all cystoscopies can be prevented at the cost of $4 \%$ of recurrences remaining undiagnosed which all were LG tumors.

\section{Discussion}

In the present study, a set of DNA methylation markers to predict the presence of bladder cancer $(\mathrm{BC})$ in urine samples has been selected. The best possible marker combination to discriminate $\mathrm{BC}$ from controls was the combination CFTR, SALL3, and TWIST1. We confirmed that these genes (and specifically CpG dinucleotides analyzed here) are hypermethylated in the bladder cancer tissue using methylation data from the TCGA Research Network [20] (Additional file 9: Figure S6). This supports their use as diagnostic markers in urine samples. In the training set, the three-gene methylation classifier achieved an AUC 0.874 while in the testing set, an AUC 0.741 was achieved to discriminate recurrent from non-recurrent patients in follow-up for bladder cancer (PFBC). These results improved significantly in 


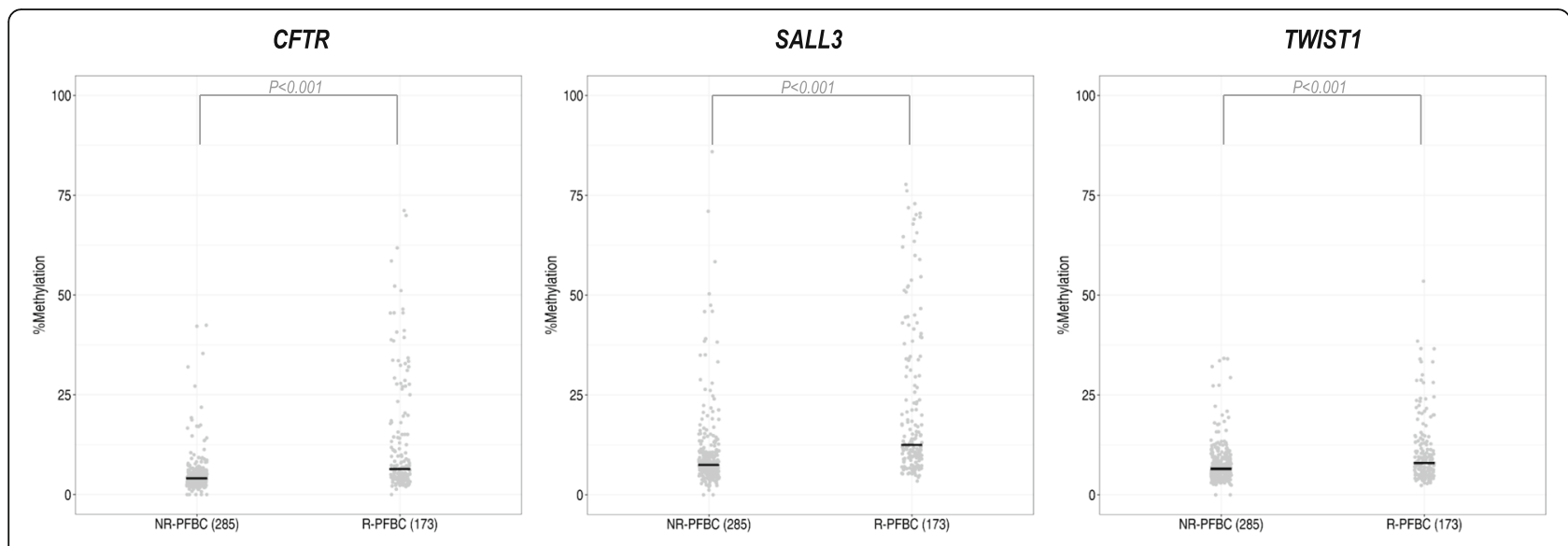

Fig. 3 Percentage of DNA methylation in recurrent and non-recurrent PFBC urine sediments for the three genes of the classifier in the validation phase. The number of samples in each group is given in brackets. Abbreviations: NR-PFBC, non-recurrent patients in follow-up for bladder cancer; R-PFBC, recurrent patients in follow-up for bladder cancer

the testing set when cytology results were included in the analysis (AUC 0.86).

TWIST1 hypermethylation in $\mathrm{BC}$ was first described by Renard and co-workers [16]. In a case-control study ( $n=145$ cases/321 controls), they detected TWIST1 and NID2 hypermethylation in urine sediments of $\mathrm{BC}$ patients using methylation-specific PCR. This two-gene panel achieved a SN of $90 \%$, SP of $93 \%$, PPV of $86 \%$, and NPV of 95\%. Nevertheless, the group of Fantony published conflicting results. They found only a $\mathrm{SN}$ of $67 \%$ and SP of $69 \%$ for this two-gene urine panel
[21]. However, the results were significantly better in the subgroup of active smokers. Unfortunately, we did not collect information about tobacco smoking, and therefore, we cannot perform a subset analysis for smoking behavior.

$\mathrm{Yu}$ and co-workers previously found in a case-control study that CFTR and SALL3, out of 59 genes that were screened, were the most frequently methylated genes to predict the presence of $\mathrm{BC}$ in urine [15]. However, in this study, no PFBC were included. In daily clinical practice, this group is especially of interest. It is not very
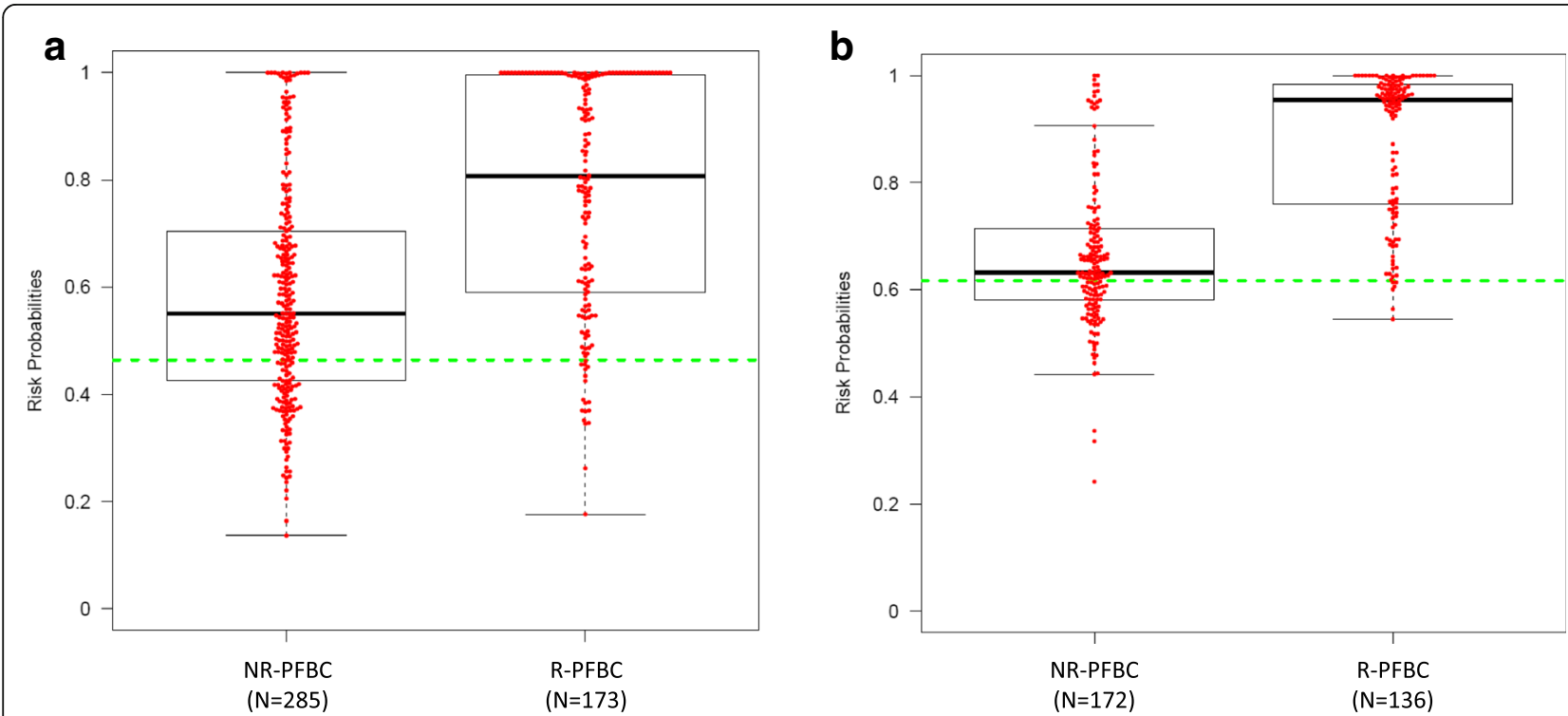

Fig. 4 Box plots showing the individual risk probabilities derived from (a) the three-gene methylation classifier and (b) the combined three-gene methylation/cytology classifier for recurrent and non-recurrent PFBC in the cross-sectional study. Dots above the cutoff value (dashed line) denote positive samples, whereas those below signify negatives scores. Cutoff values for the three-gene methylation classifier and the combined three-gene methylation/cytology classifier are $\geq 0.464$ and $\geq 0.617$, respectively. Abbreviations: NR-PFBC, non-recurrent patients in follow-up for bladder cancer; R-PFBC, recurrent patients in follow-up for bladder cancer 
likely that biomarkers will replace ureterocystoscopy in patients with macroscopic hematuria referred to the urologist. But in PFBC, urinary biomarkers with a high SN and high NPV could make a difference, i.e., lower the number of follow-up cystoscopies. Our combined three-gene methylation/cytology classifier achieves a high SN and NPV, for both high-risk and non-high-risk NMIBC. Consequently, more than a third of all cystoscopies could be prevented.

The SP of the combined classifier in the testing set, using PFBC samples, is expected to be lower than the SP of the training set, using $\mathrm{BC}$ and control samples. Possible reasons for methylation observed in PFBC samples are small tumors not yet detected by cystoscopy, residual tumor cells at the resection site, or epigenetically changed urothelial cells at the resection site or else in the bladder also known as epigenetic field defect [22]. In patients with persistent hypermethylation, which does not recur within 18 months, the presence of an epigenetic field defect is most likely. Wolff and co-workers suggested that the aberrant methylation is caused by a generalized epigenetic alteration in the whole bladder urothelium, and this widespread methylated urothelium may be the cause of the high recurrence rate in NMIBC [22].

The discovery of highly sensitive methylation markers allows us to lower the number of follow-up cystoscopies in more than a third of all follow-up patients. In the clinical situation, patients supply a urine sample before cystoscopy to perform the test. If the combined test is positive, patients will undergo a cystoscopy. In our validation series, $60 \%$ of NR-PFBC had a positive combined test and should undergo a cystoscopy. This is not a major problem since in normal daily practice, follow-up patients would have undergone a cystoscopy anyway. If the combined test is negative, cystoscopy could be skipped.

However, a methylation test has also financial and logistic implications, which means that a cost-effectiveness analysis is necessary. Using our three-gene methylation/ cytology classifier, $4 \%$ of PFBC are wrongly diagnosed as not having a recurrence; all of them had LG NMIBC. Of note, cystoscopy, our gold standard, misses up to $15 \%$ of the papillary and up to $30 \%$ of the flat lesions [4].

The strengths of this study lie in the fact that we have chosen for a two-stage approach using PFBC in the validation phase. Furthermore, the use of voided urine samples to analyze the DNA methylation status allows the development of a non-invasive $\mathrm{BC}$ diagnostic tool with an easy translation into clinical practice. However, some limitations should be mentioned. To avoid inefficiency, patients with a recurrence were oversampled by also recruiting patients who were scheduled for a TURBT of a proven bladder tumor. Consequently, the number of NR-PFBC was misrepresented in the validation series, and we had to make an estimation to calculate the number of cystoscopies that could be skipped. Secondly, $13 \%$ of the samples had to be excluded due to technical failures. Thirdly, intravesical treatments in NMIBC patients may have influenced methylation patterns. Finally, we have evaluated only a limited number of hypermethylated genes with diagnostic value previously described in the literature.

\section{Conclusions}

In conclusion, this combined three-gene methylation/cytology classifier can reduce the number of follow-up cystoscopies in PFBC. This approach may improve the patients' quality of life. For a definitive conclusion, replication of the classifier in another series of patients and cost-effectiveness studies are needed.

\section{Additional files}

Additional file 1: Figure S1. Flowchart of the entire study. A total of seven hypermethylated genes, differentially expressed between BC patients and controls $(n=168)$, were determined in the discovery phase. With these results, a three-gene methylation classifier was developed. This three-gene classifier was tested in a cross-sectional study (validation phase; $n=458$ ). Samples with available cytology results in each phase are indicated. Abbreviations: BC, bladder cancer; C, control; R-PFBC, recurrent patients in follow-up for bladder cancer; NR-PFBC, non-recurrent patients in follow-up for bladder cancer. (PPTX $75 \mathrm{~kb}$ )

Additional file 2: Table S1. Primer sequences used in PCR and pyrosequencing. (DOCX $13 \mathrm{~kb}$ )

Additional file 3: Figure S2. Pearson correlation coefficient heat map of the percentage of methylation for every $\mathrm{CpG}$ dinucleotide in the seven genes. Every CpG sites are correlated (via the Pearson correlation) with all others. Correlations are scaled by the color of the corresponding cell. Parameters are represented in the same order on the $x$ - and $y$-axes. (PPTX 1232 kb)

Additional file 4: Figure S3. Representative pyrograms showing gene methylation patterns in DNA urine samples from a bladder cancer patient. Percentage of methylation is indicated above peaks (gray columns) corresponding to the CpG sites in this region. (PPTX $183 \mathrm{~kb}$ )

Additional file 5: Table S2. Percentage of methylation for each CpG dinucleotide in the seven selected genes in control and bladder cancer urine samples. Underlined in grey the CpG site used for methylation analysis. Abbreviations: SDV; Standard Deviation. (DOCX 21 kb)

Additional file 6: Table S3. Diagnostic performance of the three-gene methylation classifier, cytology, and the combined methylation/cytology classifier in the training and testing subset of samples with cytology available. Abbreviations: LG, Low Grade; HG, High Grade; AUC, area under curve; MIBC, muscle invasive bladder cancer; NMIBC, Non-Muscle Invasive Bladder Cancer; NPV, Negative Predictive Value; PPV, Positive Predictive Value; SN, Sensitivity; SP, Specificity; BC, Bladder Cancer; C, Control; RPFBC, Recurrent Patients in Follow up for Bladder Cancer; NR-PFBC, Non Recurrent Patients in Follow up for Bladder Cancer. (DOCX 20 kb)

Additional file 7: Figure S4. Sensitivity, negative and positive predictive values of urine cytology, the three-gene methylation classifier, and the combined three-gene methylation/cytology classifier in the testing set ( $N=$ 308). Overall specificity was $94 \%$ for urine cytology, $27 \%$ for the three-gene methylation classifier, and $40 \%$ for the combined three-gene methylation/ cytology classifier. Abbreviations: LG, low-grade; HG, high-grade; NMIBC nHR, non-muscle-invasive bladder cancer non-high risk; NMIBC HR, non-muscle-invasive bladder cancer high risk; NPV, negative predictive value; PPV, positive predictive value. (PPTX $189 \mathrm{~kb}$ ) 
Additional file 8: Figure S5. Flow diagram of participants in the cross-sectional study according a) to the three-gene methylation classifier and cytology results and b) to the combined three-gene methylation/cytology classifier. Abbreviations: R-PFBC, recurrent patients in follow-up for bladder cancer; NR-PFBC, non-recurrent patients in follow-up for bladder cancer; Cytol, cytology; NA, non-available; Test, combined three-gene methylation/cytology classifier. (PPTX 85 kb)

Additional file 9: Figure S6. DNA methylation profiles for bladder cancer and control tissue samples for the three-gene classifier. Data obtained from Wanderer Web page: http://maplab.imppc.org/wanderer/. The red arrow indicates the $\mathrm{CpG}$ dinucleotide analyzed in each of the three genes. (PPTX $203 \mathrm{~kb}$ )

\section{Abbreviations}

AUC: Area under the curve; BC: Bladder cancer; BPH: Benign prostatic hyperplasia; C: Control; CIS/Tis: Carcinoma in situ; HG: High-grade; HR: High-risk; LG: Low-grade; MIBC: Muscle-invasive bladder cancer; NA: Not available; nHR: Non-high risk; NMIBC HR: Non-muscle-invasive bladder cancer high risk; NMIBC nHR: Non-muscle-invasive bladder cancer non-high risk; NMIBC: Nonmuscle-invasive bladder cancer; NPV: Negative predictive value; NR-PFBC: Nonrecurrent patients in follow-up for bladder cancer; PPV: Positive predictive value; R-PFBC: Recurrent patients in follow-up for bladder cancer; SN: Sensitivity; SP: Specificity; TURBT: Transurethral resection of the bladder tumor

\section{Acknowledgements}

We thank the technical support from the staff of the Servei Veterinari de Genètica Molecular, Facultat de Veterinària, Universitat Autònoma de Barcelona. Part of the work was developed at the building Centre de Recerca Biomèdica Cellex, Barcelona. Furthermore, funding from CERCA Programme/Generalitat de Catalunya is gratefully acknowledged.

\section{Funding}

This work was supported by grants from the Dutch Cancer Society.

\section{Availability of data and materials}

Please contact the corresponding author for data requests.

\section{Authors' contributions}

AGvdH, LM, LALMK, MJR, JAW, and AA contributed to the conception and design. AGvdH, LM, MI-T, CCMvR-vdW, MB, LALMK, MJR, JAW, and AA contributed to the methodology, collection, and assembly of data. All authors contributed to the data analysis and interpretation and manuscript writing and reviewing. All authors approved the final manuscript.

\section{Ethics approval and consent to participate}

The study was approved by the Institutional Review Boards of the Hospital Clínic of Barcelona (Spain); Radboud University Medical Center in Nijmegen (The Netherlands), St. John Emergency Hospital, Bucharest (Romania); and MD Anderson Cancer Center, Houston, Texas, (USA). Patients' informed consents were obtained before the sample collection.

\section{Competing interests}

The authors declare that they have no competing interests.

\section{Publisher's Note}

Springer Nature remains neutral with regard to jurisdictional claims in published maps and institutional affiliations.

\section{Author details}

'Department of Urology Radboud University Medical Center, Nijmegen, The Netherlands. 'Laboratory and Department of Urology, Hospital Clinic of Barcelona, IDIBAPS, University of Barcelona, Barcelona, Spain. ${ }^{3} \mathrm{CIBERehd}$ Plataforma de Bioinformática, Centro de Investigación Biomédica en red de Enfermedades Hepáticas y Digestivas, Barcelona, Spain. ${ }^{4}$ Saint John Emergency Clinical Hospital, Bucharest, Romania. ${ }^{5} \mathrm{MD}$ Anderson Cancer Center, Houston, Texas, USA. ${ }^{6}$ Hospital Clínic de Barcelona, Centre de Recerca Biomèdica CELLEX, office B22, C/Casanova, 143, 08036 Barcelona, Spain.
Received: 1 December 2017 Accepted: 3 May 2018

Published online: 30 May 2018

\section{References}

1. Oosterlinck W, Lobel B, Jakse G, et al. Guidelines on bladder cancer. Eur Urol. 2002;41:105-12.

2. Fernandez-Gomez J, Madero R, Solsona E, et al. Predicting nonmuscle invasive bladder cancer recurrence and progression in patients treated with bacillus Calmette-Guerin: the CUETO scoring model. J Urol. 2009;182:2195-203.

3. Babjuk M, Bohle A, Burger M, et al. EAU Guidelines on Non-Muscle-invasive Urothelial Carcinoma of the Bladder: update 2016. Eur Urol. 2016:71:447-61.

4. Grossman HB, Gomella L, Fradet $Y$, et al. A phase III, multicenter comparison of hexaminolevulinate fluorescence cystoscopy and white light cystoscopy for the detection of superficial papillary lesions in patients with bladder cancer. J Urol. 2007:178:62-7.

5. Fradet $Y$, Grossman HB, Gomella L, et al. A comparison of hexaminolevulinate fluorescence cystoscopy and white light cystoscopy for the detection of carcinoma in situ in patients with bladder cancer: a phase III, multicenter study. J Urol. 2007;178:68-73.

6. Brown FM. Urine cytology. It is still the gold standard for screening? Urol Clin North Am. 2000;27:25-37.

7. Sherman $A B$, Koss LG, Adams SE. Interobserver and intraobserver differences in the diagnosis of urothelial cells. Comparison with classification by computer. Anal Quant Cytol. 1984;6:112-20.

8. Parker J, Spiess PE. Current and emerging bladder cancer urinary biomarkers. ScientificWorld Journal. 2011;11:1103-12.

9. Esteller M. Epigenetics in cancer. N Engl J Med. 2008;358:1148-59.

10. Saxonov S, Berg P, Brutlag DL. A genome-wide analysis of CpG dinucleotides in the human genome distinguishes two distinct classes of promoters. Proc Natl Acad Sci U S A. 2006;103:1412-7.

11. Shames DS, Minna JD, Gazdar AF. DNA methylation in health, disease, and cancer. Curr Mol Med. 2007;7:85-102.

12. Su SF, Castro Abreu AL, Chihara Y, et al. A panel of three markers hyperand hypomethylated in urine sediments accurately predicts bladder cancer recurrence. Clin Cancer Res. 2014:20:1978-89.

13. Sobin $\mathrm{LH}$, Wittekind $\mathrm{CH}$. TNM classification of malignant tumours. International union against cancer. 6th ed. New York: Wiley; 2002.

14. Lopez-Beltran A, Sauter G, Gasser T, et al. Tumours of the urinary system. In: Eble JN, Sauter G, Epstein JI, Sesterhenn IA, editors. Pathology and genetics of tumours of the urinary system and male genital organs. World Health Organization classification of tumours. Lyon: IARC Press; 2004. p. 89-157.

15. Yu J, Zhu T, Wang Z, et al. A novel set of DNA methylation markers in urine sediments for sensitive/specific detection of bladder cancer. Clin Cancer Res. 2007:13:7296-304

16. Renard I, Joniau S, van Cleynenbreugel B, et al. Identification and validation of the methylated TWIST1 and NID2 genes through real-time methylationspecific polymerase chain reaction assays for the noninvasive detection of primary bladder cancer in urine samples. Eur Urol. 2010:58:96-104.

17. Brait M, Begum S, Carvalho AL, et al. Aberrant promoter methylation of multiple genes during pathogenesis of bladder cancer. Cancer Epidemiol Biomark Prev. 2008;17:2786-94

18. Costa $\mathrm{VL}$, Henrique R, Danielsen SA, et al. Three epigenetic biomarkers, GDF15, TMEFF2, and VIM, accurately predict bladder cancer from DNAbased analyses of urine samples. Clin Cancer Res. 2010;16:5842-51.

19. Robin X, Turck N, Hainard A, et al. pROC: an open-source package for R and S+ to analyze and compare ROC curves. BMC Bioinformatics. 2011;12:77.

20. Diez-Villanueva A, Mallona I, Peinado MA. Wanderer, an interactive viewer to explore DNA methylation and gene expression data in human cancer. Epigenetics Chromatin. 2015:8:22

21. Fantony JJ, Abern MR, Gopalakrishna A, et al. Multi-institutional external validation of urinary TWIST1 and NID2 methylation as a diagnostic test for bladder cancer. Urol Oncol. 2015;33:387-6.

22. Wolff EM, Chihara Y, Pan F, et al. Unique DNA methylation patterns distinguish noninvasive and invasive urothelial cancers and establish an epigenetic field defect in premalignant tissue. Cancer Res. 2010;70:8169-78. 